\section{Hydroxychloroquine and QTc: beyond COVID-19}

\author{
Alberto Castagna, ${ }^{1}$ Francesco Vetta, ${ }^{2}$ \\ Giuseppe Attisani, ${ }^{3}$ Raffaele Costa, ${ }^{4}$ \\ Carmen Ruberto, ${ }^{1}$ Viviana Vespertini, ${ }^{5}$ \\ Lucio Cosco, ${ }^{5}$ Giovanni Ruotolo ${ }^{4}$ \\ ${ }^{1}$ Center for Cognitive Disorders and \\ Dementia, Azienda Sanitaria Provinciale \\ di Catanzaro; ${ }^{2}$ Saint Camillus \\ International University of Health \\ Sciences, Rome; ${ }^{3}$ Department of Public \\ Heath, AUSL della Romagna, Rimini; \\ ${ }^{4}$ Geriatric Unit, Pugliese-Ciaccio \\ General Hospital, Catanzaro; ${ }^{5}$ Infectious \\ Diseases Unit, Pugliese-Ciaccio General \\ Hospital, Catanzaro, Italy
}

\begin{abstract}
Hydroxychloroquine is an antimalarial drug also known for its anti-inflammatory and antiviral effects, which have raised the interest of many researchers for its potential use in COVID-19 patients. It is known that this drug, being able to influence the cardiac repolarization phase with QTc interval prolongation, can be potentially harmful, chiefly in elderly subjects with frailty syndrome, several comorbidities and polypharmacotherapy. Therefore, although electrocardiogram monitoring of QTc prolongation is the focal point for reducing the arrhythmic risk of these patients, in order to identify the most exposed patients, the traditional Comprehensive Geriatric Assessment should be combined with a multiparametric risk score for QTc prolongation.
\end{abstract}

\section{Introduction}

In December 2019, several cases of pneumonia caused by a new coronavirus, coronavirus disease 2019 (COVID-19), began to be reported. ${ }^{1,2}$ COVID-19 causes subclinical or mild disease in $85 \%$ of cases, but compared to common influenza viruses it promotes more frequent respiratory complications such as severe interstitial pneumonia, evident in $10-15 \%$ of cases. Since January 2020, COVID-19 has gradually spread to Europe and then America and the World Health Organization (WHO) has declared a pandemic status. ${ }^{3}$ Currently over 4,700,000 cases of COVID-19 have been reported worldwide, resulting in approximately 450,000 deaths. ${ }^{4}$ This disease, which mainly involves elderly patients with car- diovascular comorbidities, does not currently have treatments approved by international public health institutions. ${ }^{3}$ In this context, the use of hydroxychloroquine (HCQ), for its antiviral and anti-inflammatory effect, has progressively gained ground for the treatment of COVID-19 patients, appearing in international and national therapeutic protocols. ${ }^{5,6}$

The antiviral action of HCQ is associated with its ability to increase endosomal $\mathrm{pH}$ and interfere with the glycosylation of the severe acute respiratory syndrome-coronavirus type 2 (SARS-CoV-2) cell receptor. In addition, by inhibiting quinone reductase-2, which is involved in the biosynthesis of sialic acid (a monosaccharide acid important for the viral receptor) and inhibiting cathepsins, which leads to the autophagosome cleaving SARS-CoV-2 spike protein, this drug is recognized as a broad-spectrum antiviral agent. Indeed, some data show that HCQ is able to inhibit the entry, transport and post entry phases of SARS-CoV-2. ${ }^{7,8}$ The anti-inflammatory activity of HCQ is mainly due to an inhibition of immune activation at the cellular level, with a reduction in the signaling of Toll-like receptors, cytokine production and CD154 expression in T cells.

While preliminary results on the efficacy of HCQ in patients with COVID-19 are partial and contradictory, ${ }^{10,11}$ it is widely accepted that the use of this drug may cause side effects, especially at cardiac and ocular level. ${ }^{12,13}$ That entails Hydroxychloroquine needs adjustment based on renal function: $200 \mathrm{mg} \times 2 /$ day if eGFR $>30 \mathrm{~mL} / \mathrm{min} ; 200$ $\mathrm{mg} /$ day if $15<\mathrm{eGFR}<30 \mathrm{~mL} / \mathrm{min} ; 200 \mathrm{mg}$ every other day if $<15 \mathrm{~mL} / \mathrm{min}$ (or in 3weekly or bi-weekly dialysis). ${ }^{14,15}$

Therefore, beyond the uncertain efficacy of HCQ in COVID-19 patients, given that this drug is used in about 1.5 million patients with rheumatoid arthritis, systemic lupus erythematosus and other connective tissue disorders, the main purpose of this paper is to thoroughly examine cardiac side effects of this drug, focusing on alterations in the repolarization phase with a QTc interval prolongation. ${ }^{16}$

The electrocardiographic QT interval, more commonly referred to as the heart rate corrected QT interval or QTc, represents the ventricular action potential, i.e., the entire period between the onset of ventricular depolarization and ventricular repolarization conclusion and results from the dynamic balance of electrical currents mediated by ion channels on ventricular cardiomyocytes. ${ }^{17}$ These action potentials vary from one cell layer to another, depending on morpho-functional characteristics, influencing the dispersion of the QT interval (QTd),
Correspondence: Alberto Castagna, Center for Cognitive Disorders and Dementia DSS Catanzaro, Azienda Sanitaria Provinciale di Catanzaro, viale Crotone, 88100 Catanzaro, Italy.

Tel./Fax: +39.09617033013

E-mail: albertocastagna@tiscali.it

Key words: Hydroxychloroquine; COVID 19; QTc prolongation; ventricular arrhythmias.

Contributions: AC, FV, GA, RC, CR, VV, LC, GR, literature research and critical revisions; AC, FV, GR, wrote the paper; AC,GR, conception of the manuscript.

Conflict of interest: the authors declare no potential conflict of interest.

Ethics approval: the type of article did not use any data and the ethics committee was not necessary.

Received for publication: 27 April 2020 Revision received: 25 August 2020.

Accepted for publication: 31 August 2020

This work is licensed under a Creative Commons Attribution-NonCommercial 4.0 International License (CC BY-NC 4.0).

${ }^{\circ}$ Copyright: the Author(s), 2020

Licensee PAGEPress, Italy

Geriatric Care 2020; 6:9064

doi:10.4081/gc.2020.9064

which in turn represents a determinant of arrhythmic risk. ${ }^{18}$ A normal QTc interval is below $450 \mathrm{~ms}$ in men and $460 \mathrm{~ms}$ in women. ${ }^{17,18}$ Aging and frailty syndrome itself are closely associated with a QTc prolongation. Indeed, aging can affect the molecular determinants of the QT interval and alter the electrical balance of the myocardium through increased fibrosis processes. Moreover, aging is also associated with changes in the balance between sympathetic and parasympathetic tone that can influence myocardial repolarization and QTc duration. ${ }^{19,20}$

Literature abounds in data on genetically determined long QT syndrome and the resulting risk of sudden cardiac death, while the focus on the acquired form is still unsatisfactory, although the latter is the most common form of long QT syndrome, especially in the elderly subject. Acquired long QT syndrome is usually the result of the complex interaction between several predisposing factors such as cardiac ischemia, electrolyte imbalance, and structural heart disease. ${ }^{21,22}$

Furthermore, in the frail elderly patient, 
extra-cardiac comorbidities and the resulting polypharmacotherapy are known, but often underestimated, risk factors for the QTc interval prolongation. ${ }^{23,24}$ All of the above remarks are the basis for ensuring a prudent use of HCQ in the elderly patient, which primarily aims at the highest possible safety before efficacy.

\section{Hydroxychloroquine and QTc prolongation}

Hydroxychloroquine, in addition to a rare form of direct cardio myotoxicity, is known to promote electrical conduction impairment with AV block, bundle branch block as well as QTc interval prolongation. In fact, HCQ is structurally and mechanistically similar to the class IA antiarrhythmic quinidine, which inhibits the human ether-àgo-go related gene (hERG) potassium channel, the voltage-gated ion channel that modulates the rapid component of the delayed rectifier potassium current, IKr. ${ }^{14-16,25}$

Blocking the hERG channel lengthens ventricular repolarization, ventricular action potential duration and the surface ECG QT interval; it may also result in the reactivation of inward calcium depolarizing currents, thus promoting early postdepolarization. In the presence of a specific spatial and temporal heterogeneity in the ventricular cardiomyocyte refractoriness, early depolarizations can trigger torsade de pointes (TdP) which, although in most cases is self-terminating, in rare cases, if sustained, can degenerate into ventricular fibrillation and cause sudden cardiac death. ${ }^{21}$

Several prospective or retrospective studies have been developed in recent months on the effect of HCQ on the QTc interval in COVID-19 patients. All the scientific data agree that HCQ, alone or in combination with other drugs, e.g. azithromycin, can cause a QTc prolongation, promoting, in rare cases, threatening ventricular arrhythmias up to TdP.

A prospective study of 150 consecutive COVID-19 patients treated with HCQ 400 mg (+ azithromycin 26\%; + lopinavir-ritonavir $35 \%$; both drugs $6 \%$ ). showed, in the ECG conducted after 5 days of treatment, a QT prolongation of mild, moderate and severe $(>500 \mathrm{~ms})$ in $9 \%, 4 \%$ and $2 \%$ of patients treated respectively. ${ }^{26} \mathrm{~A}$ recent retrospective study conducted on 251 patients hospitalized with COVID-19 who received HCQ with azithromycin, showed a prolongation of QTc $>60 \mathrm{~ms}$ in $20 \%$ of patients, with TdP in one patient. ${ }^{27}$

Mercuro et al. in a retrospective study involving 90 patients hospitalized with COVID-19 who received HCQ, highlighted the role of polypharmacotherapy and the complexity of the clinical picture in promoting QTc prolongation. In fact, in addition to the finding of a greater prolongation of the QTc interval in the 53 patients receiving combined treatment with HCQ and azithromycin compared to those receiving HCQ alone, the salient data is that $19 \%$ of patients treated with HCQ alone showed a prolongation of QTc $>500 \mathrm{~ms}$, with a higher probability in those who also took loop diuretics or with more severe clinical conditions..$^{28}$

In addition, the role of multifactorial analysis in the risk assessment of QTc prolongation strongly emerges from an interesting retrospective study of COVID-19 patients treated with HCQ + azithromycin, in which all patients underwent basic ECG and continuous telemetric monitoring. ${ }^{29}$

Although this study shows that the main determinant of QTc prolongation is the use of additional drugs that could influence the ventricular repolarization phase (OR 3.69, 95\% CI 1.22-11.2), the most interesting result is that older age, cardiovascular and renal comorbidities showed an additive weight in influencing QTc prolongation.

\section{Multiparametric risk assessment of QTe prolongation}

Based on the above data, there is a strong indication of the requirement for a multiparametric QTc prolongation risk assessment model. Although not specific to older age groups, Tisdale has proposed a multifactorial evaluation model that is certainly interesting (Tisdale Risk Score = TRS). ${ }^{30,31}$ Independent predictors of QTc prolongation have been found to be:

Table 1. Tisdale Risk score for QTc interval prolongation.

Risk factor Point

\begin{tabular}{ll} 
Age $\geq 68$ years & 1 \\
Female sex & 1 \\
\hline Loop diuretic & 1
\end{tabular}

\begin{tabular}{ll} 
Serum potassium $\leq 3.5 \mathrm{mEq} / \mathrm{L}$ & 2 \\
\hline Admission $\mathrm{QTc} \geq 450 \mathrm{~ms}$ & 2
\end{tabular}

\begin{tabular}{ll} 
Acute myocardial infarction & 2 \\
\hline 2 QTc prolonging drugs* & 3
\end{tabular}

$\begin{array}{ll}\text { Sepsis } & 3 \\ \text { Hearth failure } & 3\end{array}$

One QTc prolonging drugs* 3

Maximum risk score 21

Low risk: $\leq 6$ points; Moderate risk: 7-10 points; High risk: $\geq 11$ points. *Three points for taking One QTc interval-prolonging drug; 3 additional points for taking $\geq 2$ QTc interval-prolonging drugs (for a total of 6 points). female gender, hypokalemia, a baseline QTc $>450 \mathrm{~ms}$, comorbidities such as myocardial infarction or heart failure, the administration of one or more drugs that can promote QTc prolongation, or loop diuretics. The analysis of determinants allows patients to be stratified into three risk classes: low risk ( $\leq 6$ points), moderate risk (7-10 points) and high risk ( $\geq 11$ points) (Table 1). A proper risk analysis requires a thorough knowledge of the drugs that can promote a QTc prolongation. These include not only drugs with a prevalent action on the heart such as antiarrhythmics, but also drugs that, despite having other target organs, show secondary effects on cardiac repolarization, such as antibiotics, antimycotics, anti-emetics, antipsychotics and others (Table 2). ${ }^{19,20,29}$ Recently, a retrospective longitudinal study conducted in ICU has shown high sensitivity (97\%) for TRS, which makes it useful in identifying patients at risk of developing QTc interval prolongation, however, showing a low specificity $(16 \%)$ that makes the TRS unreliable in identifying all patients at low risk of QTc interval prolongation. ${ }^{32}$

In addition, polypharmacotherapy, which is quite common in frail elderly patients, should also be considered in this respect. In fact, data from the Concord Health and Aging in Men Project have highlighted, in frail elderly subjects, the daily use of an average number of drugs $\geq 6.5$ compared to 3.5 in patients with cognitive deficit only, clearly showing the weight of the frailty syndrome in polypharmacotherapy. ${ }^{33}$ Therefore, the use of HCQ in the context of polypharmacotherapy must be judicious, analyzing, in addition to the determinants highlighted by the TRS, possible drug interactions, depending on their pharmacokinetics. Indeed, polytherapy patients show other risk factors, such as heart disease, advanced age, electrolyte abnormalities, bradycardia, kidney or liver disease. ${ }^{34}$ In fact, HCQ, like many other drugs, is substrate of Cytochrome P450 2D6 (CYP2D6), which presents a large list of drugs that can be inhibitors or inducers, thus altering both the plasma concentration and the clinical effect of many drugs, as for HCQ. ${ }^{35,36}$

Finally, Wiśniowska et al. revealed the enormous variability in the effects of drugdrug interaction which, testifying to the complexity of the problem, suggest the need to give due attention to this additional risk factor in parallel with careful monitoring of ECG before and during the administration of drugs with expected QT prolongation. ${ }^{37}$ 
Table 2. Drug associated with a known risk of QT prolongation.

\begin{tabular}{ll}
\hline Drug class & Known risk of OT prolongation \\
Anesthetic, general & Propofol, sevoflurane \\
Antiarrhythmic & Amiodarone, disopyramide dofetilide, flecainide, ibutilide, procainamide, quinidine, sotatol \\
\hline Antidepressant & Citalopram, escitalopram \\
Anticancer & Arsenic trioxide, eribulin, vandetanib \\
\hline Antiemetic & Ondansetron, droperidol \\
Antifungal & Fluconazole, pentamidine \\
\hline Antimalaria & Chloroquine, halofantrine \\
Antipsychotic & Chlorpromazine, haloperidol, pimozide, thioridazine \\
\hline Antibiotic & Azithromycin, clarithromycin, erythromycin, ciprofloxacin, levofloxacin, moxifloxacin \\
Cholinesterase inhibitor & Donepezil \\
\hline Illicit drug & Cocaine \\
Opiates & Methadone \\
\hline Phosphodiesterase 3 inhibitor & Anagrelide, cilostazol \\
\hline
\end{tabular}

Table 3. Checks to be performed in patients on hydroxychloroquine therapy to minimize the arrhythmic risk due to a QTc prolongation. Tisdale Risk Score (TRS)Periodic monitoring of the TRS, which, in any case, should be reassessed in the occurrence of clinical exacerbations or the introduction of new drugs

QTc Withhold HCQ in patients with known congenital long QT syndrome or with baseline QTc prolongation ( $\geq 500 \mathrm{~ms}$ )

QTcPeriodic ECG check of the QTc: withdraw HCQ if QTc exceeds a preset threshold of $500 \mathrm{~ms}$ or if QTc prolongation is $>60 \mathrm{~ms}$ than baseline

Cardiac rhythm Monitors cardiac rhythm, mainly in case of QTc prolongation

ElectrolytesPeriodically check the electrolytes. In case of dystonia, correct hypokalemia at $>4 \mathrm{mEq} / \mathrm{L}$, hypomagnesemia at $>2 \mathrm{mg} / \mathrm{dL}$ and hypocalcemia at $>8.5 \mathrm{mg} / \mathrm{dL}$ Drug-drug interactionsCheck possible drug-drug interactions by evaluating the pharmacokinetics of each drug

QTc prolonging drugsAvoidance of other QTc-prolonging agents whenever feasible

\section{Conclusions}

The worldwide use of HCQ, beyond COVID-19, requires a careful analysis of the risk factors for QTc prolongation in order to reduce the resulting arrhythmic risk. The simultaneous presence of several risk factors, very common in frail elderly patients with comorbidities, suggests the use of a multifactorial analysis model, in addition to the traditional comprehensive geriatric assessment. TRS appears useful for initial staging, as well as for periodic reevaluation.

However, TRS should be supplemented with further steps (Table 3): the evaluation of a possible drug-drug interaction, periodic check of electrolytes, mainly potassium, calcium and magnesium and their integration, if required. In any case, QTc ECG monitoring remains the main indicator to be monitored at regular intervals to be determined on a case-by-case basis. Therefore, while in patients with chronic HCQ use a periodic ECG and other risk factors check can be scheduled, in case of acute patients with a high risk of QTc prolongation, hospitalization with telemetric ECG check may be appropriate.

\section{References}

1. Zhu N, Zhang D, Wang W, et al. A novel coronavirus from patients with pneumonia in China, 2019. N Engl J Med 2020; 382:727-33.

2. Huang C, Wang Y, Li X, et al. Clinical features of patients infected with 2019 novel coronavirus in Wuhan, China. Lancet 2020;395:497-506.

3. Vetta F, Vetta G, Marinaccio L. Coronavirus disease 2019 (COVID-19) and cardiovascular disease: a vicious circle. J Cardiol Cardiovasc Res 2020; 1:1-12.

4. European Centre for Disease Prevention and Control. COVID-19 situation update worldwide. Available from: https://www.ecdc.europa.eu/en/geographical-distribution-2019-ncov-cases Accessed: 20 July, 2020.

5. Ornstein M, Sperber K. The antiinflammatory and antiviral effects of hydroxychloroquine in two patients with acquired immunodeficiency syndrome and active inflammatory arthritis. Arthritis Rheum 1996;39:157-61

6. Li X, Wang Y, Agostinis P, et al. Is hydroxychloroquine beneficial for
COVID-19 patients? Cell Death and Disease 2020;11:512.

7. Sinha N, Balayla G. Hydroxychloroquine and covid-19. Postgrad Med J 2020;0:1-6.

8. Devaux CA, Rolainac JM, Colsonac P, Raoultac D. New insights on the antiviral effects of chloroquine against coronavirus: what to expect for COVID-19? Int J Antimic Ag 2020;55 [Epub ahead of print].

9. Schrezenmeier E, Dörner T. Mechanisms of action of hydroxychloroquine and chloroquine: implications for rheumatology. Nature Rev Rheumatol 2020;16: 1-12.

10. Boulware DR, Pullen AS, Bangdiwala, $\mathrm{KA}$, et al. A randomized trial of hydroxychloroquine as postexposure prophylaxis for Covid-19. NEJM 2020 [Epub ahead of print].

11. Thang W, Cao Z, Han M, et al. Hydroxychloroquine in patients with mainly mild to moderate coronavirus disease 2019: open label, randomized controlled trial. BMJ 2020;369 [Epub ahead of print].

12. WHO Evidence Review Group. The cardiotoxicity of antimalarials. Malaria Policy Advisory Committee Meeting 22- 
24 March 2017, Geneva, Switzerland. Background document for Session 2.

13. Chen CY, Wang FL, Lin CC. Chronic hydroxychloroquine use associated with QT prolongation and refractory ventricular arrhythmia. Clin Toxicol (Phila) 2006;44:173-5.

14. Coppolino G, Presta P, Nicotera R, et al. COVID-19 and renal disease in elderly patients. Geriatric Care 2020;6:9029.

15. Manzo C, Gareri P, Castagna A. Psychomotor agitation following treatment with hydroxychloroquine. Drug Saf Case Rep 2017;4:6.

16. Kao LW, Furbee RB. Drug-induced q-T prolongation. Med Clin North Am 2005;89:1125-44.

17. Bohnen MS, Peng G, Robey SH, et al. Molecular pathophysiology of congenital long QT syndrome. Physiol Rev 2017; 97:89-134.

18. Yavuz B, Deveci OS, Yavuz BB, et al. QT Dispersion increases with aging. Ann Noninv Electrocardiol 2020;11:127-31.

19. Rabkin SW, Justin Cheng X-B, Thompson DJS. Detailed analysis of the impact of age on the QT interval. J Geriatr Cardiol 2016;13:740-8.

20. Ma T, Cai J, Zhu YS, et al. Association between a frailty index based on common laboratory tests and QTc prolongation in older adults: the Rugao Longevity and Ageing Study. Clin Interv Aging. 2018;13:797-804.

21. Galiano L, Hingle N, Kurlin K, et al. Mechanisms and clinical relevance of drug-induced long QT syndrome: block of hERG, drug metabolism and drug transport in the human heart. Ann Pharmacol Pharm 2020;5:1176.

22. El-Sherif N, Turitto G, Boutjdir M.
Acquired long QT syndrome and electrophysiology of torsade de pointes. Arrhythm Electrophysiol Rev 2019;8: 122-30.

23. Bo M, Ceccofiglio A, Mussi C, et al. Prevalence, predictors and clinical implications of prolonged corrected QT in elderly patients with dementia and suspected syncope. Eur J Int Med 2019; 61:34-9.

24. Vandenbriele C, Price S. QTc in the elderly. Eur Heart J 2018;39:1445.

25. Kannankeril P, Roden DM, Darbar D. Drug-induced long QT syndrome. Pharmacol Rev 2010;62:760-81.

26. Mazzanti A, Briani M, Kukavica D, et al. Association of hydroxychloroquine with QTc interval in patients with COVID-19. Circulation 2020 [Epub ahead of print].

27. Chorin E, Dai M, Shulman E, et al. The QT interval in patients with COVID-19 treated with hydroxychloroquine and azithromycin. Nat Med 2020 [Epub ahead of print].

28. Mercuro NJ, Yen CF, Shim DJ, et al. Risk of QT interval prolongation associated with use of hydroxychloroquine with or without concomitant azithromycin among hospitalized patients testing positive for Coronavirus disease 2019 (COVID-19) JAMA Cardiol 2020 [Epub ahead of print].

29. Maraj I, Hummel JP, Taoutel R, Chamoun $\mathrm{R}$, et al. Incidence and determinants of QT interval prolongation in COVID-19 patients treated with hydroxychloroquine and azithromycin J Cardiovasc Electrophysiol 2020 [Epub ahead of print].

30. Tisdale JE, Jaynes HA, Kingery JR, et al. Development and validation of a risk score to predict QT interval prolongation in hospitalized patients [published correction appears in Circ Cardiovasc Qual Outcomes. 2013 Nov;6(6):e57]. Circ Cardiovasc Qual Outcomes 2013;6:479-87.

31. Trinkley KE, Lee R, Lien H, et al. QT interval prolongation and the risk of torsades de pointes: essentials for clinicians. Curr Med Res Opin 2013;29: 1719-26.

32. Su K, McGloin R, Gellatly RM. Predictive validity of a QTc interval prolongation risk score in the Intensive Care Unit. Pharmacotherapy 2020;40:492-9.

33. Gutiérrez-Valencia $M$, Izquierdo $M$, Cesari M, et al. The relationship between frailty and polypharmacy in older people: A systematic review. Br J Clin Pharmacol 2018;84:1432-44.

34. Coppolino G, Bolignano D, Gareri P, et al. Kidney function and cognitive decline in frail elderly: two faces of the same coin? Int Urol Nephrol 2018;50:1505-10.

35. Monte AA, West K, McDaniel KT, et al. CYP2D6 genotype phenotype discordance due to drug-drug interaction. Clin Pharmacol Ther 2018;104:933-9.

36. Mascolo A, Berrino PM, Gareri P, et al. Neuropsychiatric clinical manifestations in elderly patients treated with hydroxychloroquine: a review article. Inflammopharmacology 2018;26:1141-1149.

37. Wiśniowska B, Tylutki Z, Wyszogrodzka G, Polak S. Drug-drug interactions and QT prolongation as a commonly assessed cardiac effect - comprehensive overview of clinical trials. BMC Pharmacol Toxicol 2016;17:12. 